\title{
The Millennium Galaxy Catalogue: Galaxy Bimodality
}

\author{
Simon P. Driver ${ }^{1}$, Jochen Liske ${ }^{2}$ \\ and Alister W. Graham ${ }^{3}$ \\ ${ }^{1}$ School of Physics and Astronomy, University of St Andrews, St Andrews, Scotland \\ email: spd3@st-and.ac.uk \\ ${ }^{2}$ European Southern Observatory, Karl-Schwarzschild-Str. 2, 85748 Garching, Germany \\ ${ }^{3}$ Astrophysics and Supercomputing, Swinburne University of Technology, Australia
}

\begin{abstract}
Galaxy bimodality is caused by the bulge-disc nature of galaxies as opposed to two distinct galaxy classes. This is evident in the colour-structure plane which clearly shows that elliptical galaxies (bulge-only) lie in the red compact peak and late-type spiral galaxies (discdominated) lie in the blue diffuse peak. Early-type spirals (bulge plus disc systems) sprawl across both peaks. However after bulge-disc decomposition the bulges of early-type spirals lie exclusively in the red compact peak and their discs in the blue diffuse peak (exceptions exist but are rare, e.g., dust reddened edge-on discs and blue pseudo-bulges). Movement between these two peaks is not trivial because whilst switching off star-formation can transform colours from blue to red, modifying the orbits of $\sim 1$ billion stars from a planar diffuse structure to a triaxial compact structure is problematic (essentially requiring an equal mass merger). We propose that the most plausible explanation for the dual structure of galaxies is that galaxy formation proceeds in two stages. First an initial collapse phase (forming a centrally concentrated core and black hole), followed by splashback, infall and accretion (forming a planar rotating disc). Dwarf systems coule perhaps follow the same scenario but the lack of low luminosity bulge-disc systems would imply that the two components must rapidly blend to form a single flattened spheroidal system.
\end{abstract}

Keywords. galaxies:formation, galaxies:evolution, galaxies:fundamental parameters

\section{Introduction}

The Millennium Galaxy Catalogue (Liske et al. 2003; Cross et al. 2004) contains 10095 galaxies to $B<20$ mag extending over a $37 \mathrm{deg}^{2}$ region of the equatorial sky. The redshift survey is $96 \%$ complete (Driver et al. 2005) and most galaxies have sufficient resolution to achieve reliable bulge-disc decomposition (Allen et al. 2006). We have explored galaxy bimodality in the colour-structure plane using our sample and find an extremely strong bimodal distribution in this plane (see Fig. 1 and also Driver et al. 2006). For colour we use the PSF $(u-g)_{c}$ from SDSS DR1 (Abazajian et al. 2003) and to represent structure we use the Sérsic index (Graham \& Driver 2005). Figure 1 upper left shows the observed bimodal distribution for all galaxies brighter than $M_{B}-5 \log h=-17$ mag based on the global Sérsic index. The upper right panel shows the equivalent plot but with the colour and Sérsic index now derived for the distinct bulge and disc components. In the lower panels we show the dominant stellar structures for clarity, which together define the nearby galaxy population. These are: exponential discs $(n=1)$, truncated and antitruncated discs $(n<1.2)$, and bulges $(n>1.2)$. The lack of significant overlap between the two disc populations and the bulge populations lead us to infer distinct formation mechanisms for bulge and disc systems. The coincidence of the two disk populations implies a single common disc formation process and inside out growth. 

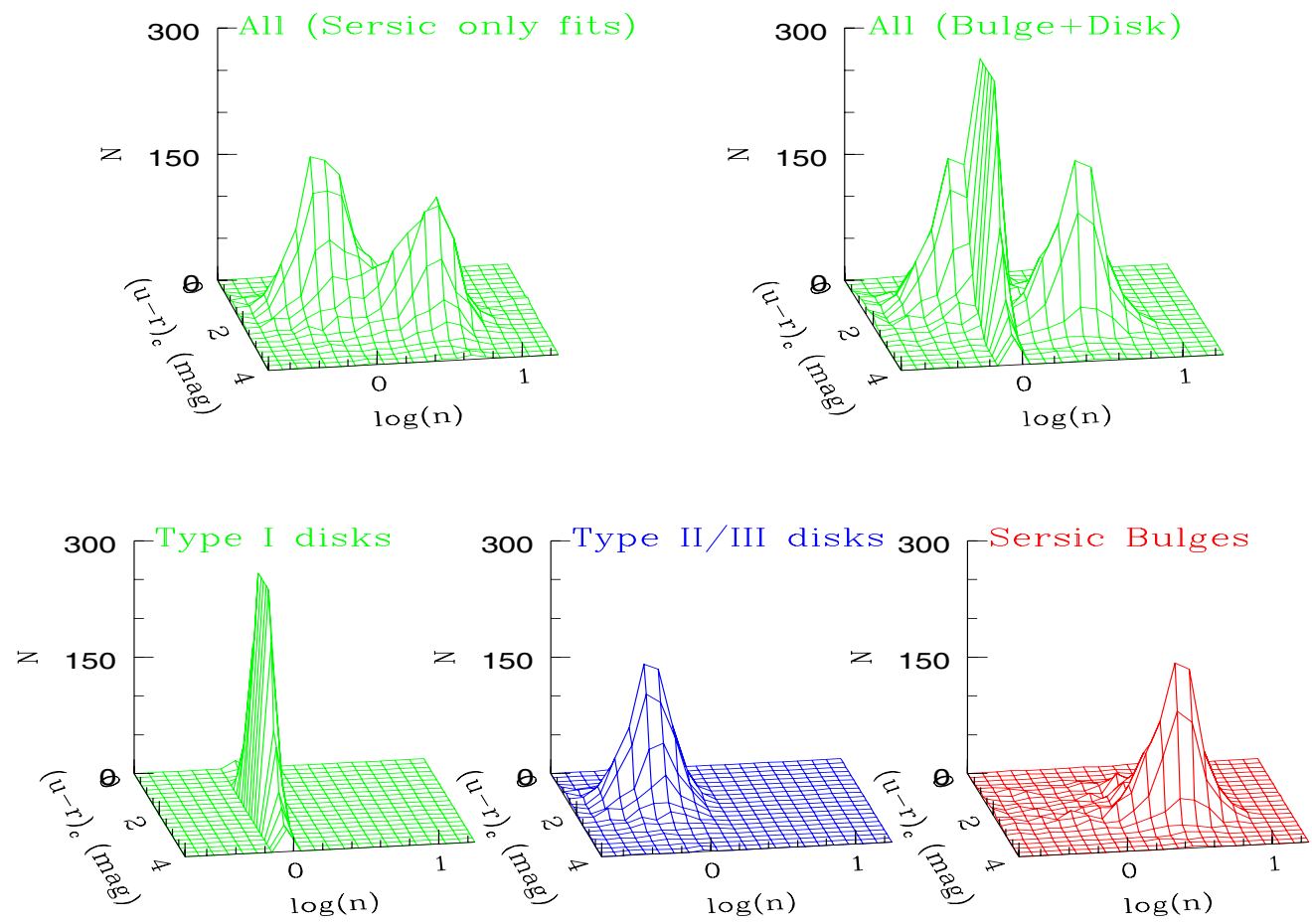

Figure 1. Galaxy bimodality in the colour-structure plane, see text for details.

\section{Conclusions}

1. Galaxies are comprised of two fundamental stellar configurations: bulges and discs.

2. Global measurements such as the star-formation rate and size evolution may be misleading (non-optimal) approaches and one should strive for distinct bulge and disc measurements instead.

3. Luminous galaxies have probably formed in two stages, early collapse to form a triaxial pressure supported bulge (with black hole), followed by splashback, infall and accretion to form a planar rotating disc.

4. Truncation of galaxy discs is common and implies ongoing inside-out growth.

5. If dwarf systems adhere to this scenario it may be that their bulge and disc components are unstable and blend. Some evidence for this is perhaps seen in the DART survey of the Fornax dwarf spheroidal (see Battaglia et al. 2006).

More details on galaxy bimodality are presented in Driver et al. (2006).

\section{References}

Allen P., et al., 2006, MNRAS, 371, 2 (astro-ph/0605699)

Abazajian K., et al., 2003, AJ, 126, 2081

Battaglia G., et al., 2006, A\&A, in press, astro-ph/0608370

Cross N.J.G., et al., 2004, MNRAS, 349, 576

Driver S.P., Liske J., Cross N.J.G., De Propris R., \& Allen P.D., 2005, MNRAS, 360, 81

Driver S.P., et al., 2006, MNRAS, 368, 414

Graham A.W., \& Driver S.P., 2005, PASA, 22, 118

Liske J., Lemon D., Driver S.P., Cross N.J.G., \& Couch W.J., 2003, MNRAS, 344, 307 